\title{
PERSONALIZED VIDEO SUMMARIZATION BASED ON GROUP SCORING
}

\author{
Kaveh Darabi, Gheorghita Ghinea \\ School of Computing and Information Systems \\ Brunel University \\ London, United Kingdom \\ \{cspgkkd1,george.ghinea\}@brunel.ac.uk
}

\begin{abstract}
In this paper an expert-based model for generation of personalized video summaries is suggested. The video frames are initially scored and annotated by multiple video experts. Thereafter, the scores for the video segments that have been assigned the higher priorities by end users will be upgraded. Considering the required summary length, the highest scored video frames will be inserted into a personalized final summary. For evaluation purposes, the video summaries generated by our system have been compared against the results from a number of automatic and semi-automatic summarization tools that use different modalities for abstraction.
\end{abstract}

\section{Index Terms-Video summarization, Personalization, Upgrading frames scores, user-centred}

\section{INTRODUCTION}

The growing amount of multimedia content has imposed the need for development of systems which are able to summarize videos of different genres automatically. Consequently, a considerable research effort has been allocated to this topic and various abstraction techniques have been developed. Broadly, two basic types of video summaries exist, static key-frames abstracts and dynamic video skims [1]. As a result of advanced audio-visual capturing tools, developing effective techniques to generate dynamic video skimming is becoming increasingly popular [2]. In an eventdriven approach, tag localization is the basis for abstraction purposes. In the first step, the tags which were associated to each video were localized into the corresponding shots. Thereafter, relevancy of each shot to the event query was assessed using the shot level tags. Finally, a set of key-shots with the highest relevance scores were identified and extracted to be included into the summary. These relevance scores were generated by analysing the iterated occurrence characteristics of key subevents [3]. However, applying these types of video summarization methods will result in generation of identical video summaries for all viewers. It is important, though, to capture the user's interests and modify the video summaries in a way that meets the user's requirements - in other words, to generate personalized video summaries. A personalized video summarization system then is designed to generate a shorter version of a video based on the user's preferences and interests while it retains the significant semantic content of the original video stream [4]. In a semi-automatic approach, [5] MPEG-7 metadata, as well as user profiling alongside a supervised learning algorithm have been the basis for generation of personalized content. In [6], a fuzzy rule-based system to approximate the human decision making Process was applied for personalized summary generation task. In [7] Human physiological responses such as respiration rate and blood volume pulse were the determining factors in extraction of personalized content. Further, in a recent research, sketches have been the basis to represent the personalized summaries of the videos using an interactive selection method for users to pre-select the available interesting objects in the video [8]. In a resource-allocation-based framework, playback speed and perceptual comfort have been the key elements for generation of personalized video summaries [9]. In this paper, we address this challenge and propose a framework to produce personalized video summaries based on video experts' assigned scores to video frames. Accordingly, the structure of this paper is as follows: Our approach is then detailed in Sections II and III, whilst Section IV presents evaluation results. Lastly, conclusions are drawn and opportunities for future work are identified in Section V.

\section{VIDEO SUMMARIZATION BY GROUP SCORING:}

In previous work [10], we have described an approach to video summarization based on a group scoring method, in which original video frames are scored by a number of video scorers (experts) and the assigned scores averaged to produce a singular value for each frame. A group of frames with the highest average scores are then chosen to be inserted into the final summary. In this approach, the required number of video experts could be varied based on the different use-case scenarios. The proposed method was evaluated and shown to achieve promising results (vis. a vis. machinegenerated approaches) in 6 different video categories. However, the generated summaries for all of the end-users were identical and their individual preferences were not envisaged in the summarization process. In this paper, we develop a model to personalize the final summaries in accordance to the individual end-user's expectations, and thus to produce a better user experience. 


\subsection{Video Segments Enrichment}

For enrichment and scoring purposes a semiautomatic model has been applied in our framework. In the first step, the original videos are segmented into a number of scenes (group of semantically and visually similar frames). Later, each scene is enriched with a group of audio and visual tags and the appointment of a representative key-frame.

\subsubsection{Scene boundary detection}

AVcutty [11] as a typical scene boundary detection tool has been adopted to determine the timestamps for each contributing scene. It should be reminded that each scene in the context of a complete video plays the same role as a paragraph in a whole text. Therefore, there should be a semantic and visual correlation and cohesion between the existing frames of a particular scene. The mentioned tool utilises the colour and motion features of the video frames for scene change detection purposes. The required minimum time length for each scene has been set to 3 seconds. Thus, any identified video scene with shorter length will be added to the next scene. This facilitates scoring and annotating of the original video by reducing the number of unnecessary pauses for the enrichment task.

\subsubsection{Video scenes annotation and scoring}

In this stage, video experts are asked to score and enrich the video segments based on the auditory, visual and textual content of the video. The video experts score the video frames 'on the fly' in a range between 0-10 using the Slider tool. Using the identified timestamps for the scene boundaries, the videos will be paused automatically at the end of each scene and the video experts immediately will be prompted to annotate the video scene using the provided graphical user interface (while the scoring process is stopped). The video scorers can optionally enrich the video scenes while the videos are halted, by assigning audio and visual tags to each scene. These tags could contain information regarding the significant events, objects and any activities in the corresponding video segment. The video scorers have the possibility to choose the previously assigned tags (by former scorers) or to add new ones based on their personal perception and priorities to the scenes. Once the annotation process for one scene is finished, the scorers will then be engaged in scoring the video frames for the following scene using the Slider tool. By re-starting the video, the initial frames from the upcoming scene are likely to be scored by unwanted grades. This is due to a predictable minor delay from the time in which video experts have to observe and evaluate the contextual significance of the opening frames (of the following scene) till the point they can actually start scoring. Therefore, to minimize the negative effect of this lag, a new pre-computed value was dynamically calculated and assigned to the Slider tool each time that a scene starts. In order to produce this value, a score was computed for each scene, by averaging the previously assigned scores from the former experts to the whole frames of that particular scene. Any recent assigned scores from new scorers will update these computed average scores.

\subsubsection{Key-Frame selection for the scenes}

During the scene enrichment stage, the annotators (experts) are also presented with a set of 3 candidate key frames at the end of each scene. The video experts are asked to elect the one that they personally perceive as the highest quality to represent and summarize the semantic and visual content of that scene. For extraction of these three nominated key frames, each video scene has to be fragmented into three equal shots in the first place, and each shot will be represented by a key frame (to improve the coverage rate of any visual content changes in whole scene). In order to select a key frame for each of these 3 identified video shots, two criteria should be considered. First, the frame has the highest assigned score between all the existing frames of that shot. Second, the candidate frame is temporally located in the middle of each shot. Therefore, between all the previously highest scored frames of each shot, the frame which is temporally closer to the centre of that shot will be introduced as a potential key frame for that video shot (to increase the likelihood of extracting more visually significant and stable frames ). These 3 nominee frames from each scene are then compared against each other from two different perspectives. Firstly, their visual content attractiveness and richness should be considered. Secondly, their capabilities in reflection of the semantic concepts of the corresponding video scene have to be taken into account. Finally, for each scene, the candidate frame that has the highest selection rate by different annotators will be selected as the representative key frame.

\subsection{Capturing The Users' Priorities}

This phase is responsible for capturing an enduser's priorities in a particular video. As a result, in prior to the generation of any final summary, the end-users will be provided with some visual and textual information regarding the content of the existing video scenes. The goal here is to prioritize the video segments based on the user's preferences and superiorities. Therefore, a list of representative key frames with their associated visual and audio tags is presented to the end users. Each of the displayed representative frames corresponds to a single video scene (these are the delegate key frames chosen by most of the video experts in the previous stage), while attached auditory and visual information to each key-frame correspond to the mostly verified tags for that scene by different video scorers (one audio content tag and one visual content tag per each scene). The end users will be asked to express their level of interest to each video scene, based on the displayed video frames and 


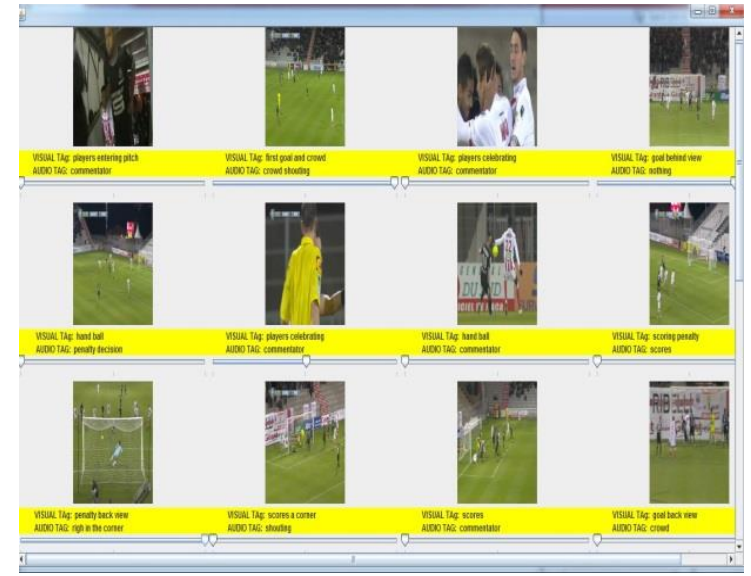

Figure 1. Interface for end-users to prioritize the scenes

tags, using the provided slider tool (Fig.1). The users could choose 3 priority levels for each scene. Level 0 has been considered for the scenes with the lowest level of significance to them, while level 1 is for the scenes with higher importance which were preferred to be included into final abstract. Level 2 designates the scenes that users found the most attractive and should be included with the highest priority into the final summary.

\subsection{Updating The Frame Scores}

In this phase, the initial generated average scores of the frames, assigned by the video scorers are updated based on the previously captured personal interests for each end-user. Therefore, based on the selected priority level for each scene by the end users, the primary average scores are updated. The scores of frames belonging to the scenes by the level 0 of interest will not be altered at all. However, in the scenes with a level 1 priority, the grades for the frames which their primary assigned scores are the highest among the frames of that scene, will be increased by 20 percent (to the maximum value of 12). This is done in order to potentially escalate the probability of incorporation of the highest quality frames of those scenes into the eventual video digest. The updated mark for the frames belonging to the scenes with the highest level of priority for a particular end-user will be recalculated in a different format. The grades for the frames which preliminary were scored the highest in each scene, will be upgraded to the maximum possible value (12). In fact, this would increase the chance of definite inclusion of the highest quality segments of those particular scenes (with level 2 priority) in the final summary. However, the marks for the frames of these scenes whose scores are not the highest but nonetheless manage to exceed the respective scene's average scores will be boosted by 20 percent as well (to the maximum of 12). The scores for the remaining frames of these scenes will remain unchanged.

\section{GENERATING THE PERSONALIZED SUMMARY}

In the final step, the personalized video summaries are produced based on the updated frames scores. In accordance to the summarization method based on group scoring, [10] the highest scored frames alongside the audio and textual content are selected and inserted into the final video digest. Considering the required number of frames, those highest scored frames will be selected to be added to a final list and to be sorted based on their time order in the original video. ReqNO calculates the required number of frames for extraction while TarVidTime shows the required video summary time.

ReqNO = TarVidTime( seconds)

$\times$ FramesFrequencyScale

So, if $\mathrm{K}$ represents the frame number in the original video, $\mathrm{L}$ is a list of chosen frames.

$$
\begin{aligned}
& L=\left\{\mathrm{F}_{\mathrm{K}} \mid 0<K<\right.\text { ReqNO \&AvgFra } \\
& \left.\geq \text { AvgFrame }_{\mathrm{U}_{\mathrm{i}=1}^{\mathrm{N}-\mathrm{ReqNo}^{\prime}} \mathrm{L}^{\prime}(\mathrm{i})}\right\} \\
& \text { SortedFrames }=\left\{F_{j} \mid 0<j<\text { ReqNo \& } T_{F_{j}}>T_{F_{j-1}}\right\}
\end{aligned}
$$

Using this sorted list, the temporally corresponding audio and text segments with those elected frames will be copied from the original tracks into the summary video. Considering that semantically and temporally close frames are usually similarly scored, the number of sudden cuts in the generated summary could drop significantly and video consistency and continuity are improved. As a result, more meaningful auditory and visual contents can be included in the final digest.

\section{EXPERIEMENTS AND EVALUATION}

A group of short videos (2 minutes each) from 6 different video categories comprising, Movie, Sport, Documentary, Advertisement, Music and News genres were used to investigate the effectiveness of the proposed approach. 10 operators (video experts) with different demographic details (5 Female and 5 Male within age range of 25-45) were asked to watch each of these 6 videos and to score and enrich the different segments of the videos based on their personal perceptions and preferences. As was mentioned in the last section, the experts have the option to select the previously assigned tags or to skip the annotation stage. However, they had to score the frames and to choose the representative key frame of each scene. The assigned scores for each frame were then averaged to generate a singular value for that frame. In order to produce personalized summaries, we adopted 30 end-users (15 Female and 15 Male within the age range of 20-60) to understand their priorities towards different scenes within the original videos based on the proposed method in section 2.2. These users were of course different to the 10 experts who scored the videos initially. 


\subsection{Analysis Of The Generated Summaries}

In order to assess the quality of our personalized video summarization approach, the generated results have been compared against the video abstracts produced by 4 other systems. 3 of these tools summarize the videos automatically by assessment of different modalities and applying statistical and mathematical algorithms while the fourth tool, functions semi-automatically based on human involvement. The 6 original videos alongside their 5 summary versions created by 5 existing tools (including the personalized summaries generated for each specific user using our proposed technique) were presented to the same 30 end-users on the basis of whose inputs their personalized summaries were created. These 5 summaries from each category were shown to the users in a random order so as to minimize order effects. Moreover, no information regarding the corresponding adopted summarization tools for each of the summary versions was revealed to participants. After watching the original video and the summaries the users were asked to score each of the generated abstracts awarding marks between 0 (worst video summary possible) to 10 (best video summary possible), from 4 different perspectives consisting of Recall (Re), Precision (Pe), Timing (Ti) and Overall Satisfaction (OS).These measures were described in details in our previous work[10]. The given scores for each of these measures were averaged over 30 users and their mean values for each of the video categories are given in Table I. S1[12], S2 [13], S3 [14], S4 [10] and S5 indicate the average achieved scores by, respectively, the first, second, third, fourth and our recent proposed personalized systems

\subsection{Validation Of The Statistical Results}

Our proposed method has been scored highest from the Precision and Overall satisfaction point of views across all 6 existing categories. High Precision scores can justify the effectiveness of our method in producing the personalized results. As it can indicate that the video segments with higher priorities to each individual end-user have been identified to be inserted into the final digest considerably. Our model managed to deliver the best quality video digest among all 6 categories based on the average Overall Satisfaction marks. In order to validate the statistical significance of the assigned scores for our new proposed tool a t-test analysis has been adopted. These two main indicators were compared pairwise against the achieved scores by the other 4 systems and the results are displayed in Table II. The outcome of this test highlights statistically significant differences (at the $\mathrm{p}=0.05$ level) between the scored obtained by S5 (our new tool) and the other 4 summarization systems across these two measures. Generally, the S1 tool generates some good results in terms of Recall and Precision, however, the nature of this method leads to lower grades in terms of Overall Satisfaction. Summarizing the audio and video tracks separately and concatenation of static key-frames to generate slide shows thus have a negative effect on the general experience of endusers. The second method could achieve some good results for particular categories including the Movie and Music Video. However, the performance is considerably domain-dependent. The results for the fourth system enjoy acceptable user ratings over 6 different categories. However, lower scores for Precision and Overall Satisfaction are due to the inability of this method to actually generate personalized content.

\section{CONCLUSION AND FUTURE WORK}

In this paper, a new method for producing personalized video summaries has been proposed. Experimental results indicate the effectiveness of this approach in delivering superior outcomes comparing to our previously proposed method and 3 other automatic summarization tools. However, proposing a method which requires a less end-user involvement is a topic for our future work.

TABLE I. EVALUTAION OF PROPOSED TOOL AGAINST THE OTHER 4 TOOLS

\begin{tabular}{|c|c|c|c|c|c|c|c|c|c|c|c|c|c|c|c|c|c|c|c|c|}
\hline & \multicolumn{4}{|c|}{ S1 } & \multicolumn{4}{|c|}{$\mathrm{S} 2$} & \multicolumn{4}{|c|}{ S3 } & \multicolumn{4}{|c|}{ S4 } & \multicolumn{4}{|c|}{ S5 } \\
\hline & $\mathrm{Re}$ & $\mathrm{P}_{i}$ & $\mathrm{Ti}$ & $\mathrm{OS}$ & $\mathrm{Re}$ & $\mathrm{P}_{i}$ & $\mathrm{Ti}$ & OS & $\mathrm{Re}$ & $\mathrm{P}_{\mathrm{i}}$ & $\mathrm{Ti}$ & OS & $\mathrm{Re}$ & $\mathrm{P}_{i}$ & $\mathrm{Ti}$ & OS & $\mathrm{Re}$ & $\mathrm{P}_{1}$ & $\mathrm{Ti}$ & $\mathrm{OS}$ \\
\hline MOV & 7.8 & 7.6 & 9.1 & 4.1 & 7.0 & 7.5 & 7.8 & 6.3 & 4.3 & 4.4 & 6.5 & 4.0 & 7.1 & 6.8 & 10 & 7.2 & 6.5 & 8.3 & 10 & 7.9 \\
\hline ADV & 7.5 & 7.7 & 9.0 & 3.9 & 6.0 & 5.6 & 72 & 5.4 & 6.8 & 6.5 & 63 & 4.1 & 7.7 & 8.2 & 10 & 7.8 & 7.5 & 8.7 & 10 & 8.3 \\
\hline $\mathrm{DOC}$ & 7.7 & 7.1 & 9.1 & 4.3 & 7.3 & 6.9 & 79 & 5.8 & 5.1 & 6.1 & 6.7 & 4.5 & 6.7 & 7.1 & 10 & 7.2 & 6.8 & 79 & 10 & 8.0 \\
\hline NEW & 4.3 & 6.7 & 8.6 & 2.0 & 6.1 & 5.8 & 7.7 & 3.4 & 5.3 & 5.1 & 5.9 & 1.9 & 6.4 & 6.7 & 10 & 6.1 & 6.6 & 7.5 & 10 & 7.1 \\
\hline SPO & 6.9 & 6.0 & 8.3 & 3.4 & 5.8 & 5.8 & 7.8 & 5.4 & 4.5 & 3.8 & 5.7 & 4.1 & 6.9 & 7.4 & 10 & 6.9 & 6.5 & 7.8 & 10 & 7.4 \\
\hline MUS & 7.7 & 6.8 & 8.5 & 3.1 & 6.8 & 6.4 & 79 & 5.4 & 5.8 & 5.7 & 6.2 & 3.5 & 6.5 & 6.8 & 10 & 6.3 & 6.2 & 7.6 & 10 & 7.2 \\
\hline
\end{tabular}

TABLE II. STATISTICAL TEST ANALYSIS FROM THE PRECISION AND OVERALL SATISFACTION POINTS OF VIEW

\begin{tabular}{|c|c|c|c|c|c|c|c|c|c|c|c|c|c|c|c|c|}
\hline & \multicolumn{4}{|c|}{ S5-S4 } & \multicolumn{4}{|c|}{$\mathrm{S} 5-\mathrm{S} 3$} & \multicolumn{4}{|c|}{$\mathrm{S} 5-\mathrm{S} 2$} & \multicolumn{4}{|c|}{$\mathrm{S} 5-\mathrm{S} 1$} \\
\hline & \multicolumn{2}{|c|}{$\mathrm{Pe}$} & \multicolumn{2}{|c|}{$\mathrm{OS}$} & \multicolumn{2}{|c|}{$\mathrm{Pe}$} & \multicolumn{2}{|c|}{ OS } & \multicolumn{2}{|c|}{$\mathrm{Pe}$} & \multicolumn{2}{|c|}{ OS } & \multicolumn{2}{|c|}{$\mathrm{Pe}$} & \multicolumn{2}{|c|}{$\mathrm{OS}$} \\
\hline & $\mathrm{T}$ & $\mathrm{P}$ & $\mathrm{T}$ & $\mathrm{P}$ & $\mathrm{T}$ & $\mathrm{P}$ & $\mathrm{T}$ & $\mathrm{P}$ & $\mathrm{T}$ & $\mathrm{P}$ & $\mathrm{T}$ & $\mathrm{P}$ & $\mathrm{T}$ & $\mathrm{P}$ & $T$ & $\mathrm{P}$ \\
\hline SPO & 2.3 & 0.012 & 2.34 & 0.025 & 13.88 & 1.2E-14 & 15.14 & $1.3 \mathrm{E}-15$ & 7.65 & $9.04 E-9$ & 6.02 & $7.5 \mathrm{E}-7$ & 3.23 & 0.0015 & 1287 & $8.07 \mathrm{E}-14$ \\
\hline DOC & 3.18 & 0.0017 & 3.37 & 0.0010 & 5.57 & $2.5 E-6$ & 13.25 & $3.8 \mathrm{E}-14$ & 3.65 & 0.0004 & 593 & $9.6 \mathrm{E}-7$ & 2.11 & 0.021 & 15.00 & $1.6 \mathrm{E}-15$ \\
\hline NEW & 3.31 & 0.0012 & 4.11 & 0.0001 & 6.39 & $3.5 E-7$ & 1493 & $1.7 \mathrm{E}-15$ & 496 & $1.3 \mathrm{E}-5$ & 11.45 & $1.2 \mathrm{E}-12$ & 206 & 0.024 & 16.5 & $1.2 \mathrm{E}-16$ \\
\hline ADV & 2.46 & 0.009 & 2.64 & 0.006 & 6.89 & $7.1 \mathrm{E}-\mathrm{s}$ & 125 & $1.4 \mathrm{E}-13$ & 10.54 & $5.0 \mathrm{E}-12$ & 8.02 & 3.7E-9 & 4.25 & $99 \mathrm{E}-5$ & 11.67 & $8.7 E-13$ \\
\hline MUS & 4.32 & $8.2 \mathrm{E}-5$ & 3.88 & 0.0002 & 5.4 & $4.1 \mathrm{E}-6$ & 1251 & $1.6 \mathrm{E}-13$ & 3.19 & 0.0016 & 6.22 & $4.3 \mathrm{E}-7$ & 2.10 & 0.022 & 991 & $3.9 \mathrm{E}-11$ \\
\hline $\mathrm{MOV}$ & 4.96 & $1.4 \mathrm{E}-5$ & 2.91 & 0.0034 & 12.64 & $1.2 \mathrm{E}-13$ & 10.14 & $2.3 \mathrm{E}-11$ & 2.14 & 0.0203 & 4.05 & 0.00017 & 2.15 & 0.0196 & 11.03 & $3.3 \mathrm{E}-12$ \\
\hline
\end{tabular}




\section{REFERENCES}

[1] W. Ren and Y. Zhu, "Video summarization approach based on machine learning", IEEE, Intelligent Information Hiding and Multimedia Signal Processing, pp.450-453, August 15-2009

[2] X. Li, "Image Annotation by Large Scale Content Based Image Retrieval", Proc. ACM Int'l Conf. Multimedia, pp. 607-610, 2006.

[3] M. Wang, R. Hong, G. Li, Z. Zha, S. Yan and T. Chua, "Event Driven Web Video Summarization by Tag Localization and Key-Shot Identification," Multimedia, IEEE Transactions on , vol.14, no.4, pp.975,985, Aug. 2012

[4] Y. Takahashi, N. Nitta and N. Babaguchi, "Automatic Video Summarization of Sports Videos Using Metadata", Advances in Multimedia Information Processing, Vol. 3332, pp. 272-280, 2005

[5] A. Jaimes, T. Echigo, M. Teraguchi and F. Satoh, "Learning personalized video highlights from detailed MPEG-7 metadata," Image Processing. 2002. Proceedings. 2002 International Conference on , vol.1, no., pp.I-133,I-136 vol.1, 2002

[6] H. Park and S. Cho, "A personalized summarization of video life-logs from an indoor multicamera system using a fuzzy rule-based system with domain knowledge", Information Systems, Volume 36, Issue 8, Pages 1124-1134, December 2011

[7] A. Money and H. Agius, "Analyzing User Physiological Responses for Affective Video Summarization." Displays (Elsevier), vol. 30, no 2, pp. 59-70, 2009

[8] Y. Zhang,C. Ma, J. Zhang, D. Zhang, and Y. Liu," An interactive personalized video summarization based on sketches". In Proceedings of the 12th ACM SIGGRAPH International Conference on Virtual-Reality Continuum and Its Applications in Industry (VRCAI '13). ACM, New York, USA, pp. 249-258, 2013

[9] F. Chen, C. Vleeschouwer and A. Cavallaro, "Resource Allocation for Personalized Video Summarization," Multimedia, IEEE Transactions on, vol.16, no.2, pp.455-469, Feb. 2014

[10] K. Darabi and G. Ghinea, "Video summarization based on group scoring", In proceeding of the $4^{\text {th }}$ IEEE International Conference on Multimedia computing and Systems, Marrakech, 2014, PP. xxx-xxx

[11] http://www.avcutty.de/english/ (Accessed 25 December 2013)

[12] J. You, M. Hannuksela and M. Gabbouj, "Semantic audio-visual analysis for video summarization", IEEE Region 8 EUROCON 2009 Conference, 2009

[13] G. Evangelopoulos, A. Zlatintsi, A. Potamianos, P. Maragos , K. Rapantzikos, G. Skoumas and Y. Avrithis, "Video Event Detection and Summarization Using Audio, Visual and Text Saliency", Proceeding of IEEE Int'l Conf. on Acoustics, Speech and Signal Processing (ICASSP-09), Taipei, Taiwan, Apr. 2009

[14] M. Beom, L. Williem, and I. Park, "Spatiotemporal Saliency-Based Video Summarization on a Smartphone", JBE, vol. 18, no. 2, pp.185-195, March 2013 